AperTO - Archivio Istituzionale Open Access dell'Università di Torino

\title{
Advanced visual interfaces for cultural heritage
}

\section{This is the author's manuscript}

Original Citation:

\section{Availability:}

This version is available http://hdl.handle.net/2318/1617593

since 2016-11-28T15:39:17Z

Publisher:

Association for Computing Machinery

Published version:

DOI:10.1145/2909132.2927470

Terms of use:

Open Access

Anyone can freely access the full text of works made available as "Open Access". Works made available under a Creative Commons license can be used according to the terms and conditions of said license. Use of all other works requires consent of the right holder (author or publisher) if not exempted from copyright protection by the applicable law. 


\title{
Advanced Visual Interfaces for Cultural Heritage
}

\author{
2016 Workshop Summary
}

\author{
Cristina Gena \\ The University of Turin \\ Corso Svizzera 185 \\ Italy, 10149 \\ $+390116706827$
}

cristina.gena@unito.it

\author{
Berardina De Carolis \\ The University of Bari \\ "Aldo Moro" \\ Italy, 70126 \\ $+390805443284$
}

berardina.decarolis@uniba.it

\author{
Tsvi Kuflik \\ The University of Haifa \\ Mount Carmel, Haifa, \\ Israel, 31905 \\ +97248288511 \\ tsvikak@is.haifa.ac.il
}

\author{
Fabrizio Nunnari \\ DFKI \\ Campus D3.2, 66123 \\ Saarbrücken, Germany \\ +496813023393 \\ fabrizio.nunnari@dfki.de
}

\begin{abstract}
Cultural heritage traditionally draws a lot of research attention. The domain is rich of physical as well as virtual sites and infinite information about items. Hence, it is only natural that whenever new technology appears, it is experimented in cultural heritage - from early dialog systems to state of the art Humanoid robots, eye tracking, virtual/augmented reality, and the Internet of Things (IoT). The AVI-CH workshop, in the diversity of topics presented by the accepted papers, nicely demonstrate this.
\end{abstract}

\section{Author Keywords}

Advanced Visualization, Cultural Heritage, Workshop.

\section{ACM Classification Keywords}

Human-centered computing - Human computer interaction (HCI)

\section{INTRODUCTION}

The rapid development of information and communication technologies (ICT) and the Internet has enabled cultural heritage $(\mathrm{CH})$ institutions to provide access to their collections in multiple various ways, both on-site and online, and to attract even wider audiences than those that visit the physical museums.

In parallel and part of the above, there is an enormous growth in user interfaces and in information visualization technologies. The range of interfaces is growing by the day from tiny smart watch screens to wall-size large public displays.

Regarding virtual advanced interfaces, there are several successful examples of 3D technologies for virtual museums. The use of (web) 3D in cultural heritage promotion allows the general public to live immersive experiences in virtual, reconstructed locations, like ancient towns and locations, and to visit existent, but remotely located locations, such as world-

SAMPLE: Permission to make digital or hard copies of all or part of this work for personal or classroom use is granted without fee provided that copies are not made or distributed for profit or commercial advantage and that copies bear this notice and the full citation on the first page. To copy otherwise, or republish, to post on servers or to redistribute to lists, requires prior specific permission and/or a fee.

Conference'10, Month 1-2, 2010, City, State, Country. Copyright 2010 ACM 1-58113-000-0/00/0010 _..\$15.00.

DOI: http://dx.doi.org/10.1145/12345.67890 wide cultural institutions (such as Google Art Project [1]). For preservation purposes, web $3 \mathrm{D}$ provides scholars and cultural heritage professionals with a way to consult and maintain visual repositories of real exhibits, with the possibility of visualizing, comparing and studying 3D digital equivalents of real artworks physically situated in different locations.

Cultural heritage is one challenging domain of application for such novel ICT technology. $\mathrm{CH}$ is ubiquitous - just look around you. There is abundance of $\mathrm{CH}$ related information available, about almost every object we can think of. How can we access and enjoy this information in Ubiquitous Computing scenario?

Advanced and natural human-computer interaction is a key factor in enabling such access and visual interfaces, whether they are tiny mobile screens or large wall mounted displays, they can all be part of the $\mathrm{CH}$ IoT and be part of a ubiquitous $\mathrm{CH}$ infrastructure, where information can be personalized and displayed/projected, on screens or overlaid on real objects.

The goal of the workshop is to bring together researchers and practitioners interested in exploring the potential of state of the art, advanced visual interfaces in enhancing our daily cultural heritage experience.

\section{SUBMISSIONS}

The 13 submissions that were accepted spanned a large variety of topics combining advanced visual interfaces and cultural heritage. We discuss these submissions from several aspects, first of all looking at onsite vs. online approaches and then at the variety of interaction techniques and technologies involved.

\subsection{On site interaction}

Relatively large and diverse number of the submissions focused on supporting the museum visitor during the visit, applying variety of techniques for different applications. Partarakis et al. [11] presents the design and implementation of a technological framework based on Ambient Intelligence to enhance visitor experiences within Cultural Heritage Institutions (CHIs) by augmenting two-dimensional paintings. Among the major contributions of this research work is the support of personalized multi-user access to exhibits, facilitating also adaptation mechanisms for altering the 
interaction style and content to the requirements of each CHI visitor. A standards compliant knowledge representation and the appropriate authoring tools guarantee the effective integration of this approach to the CHI context. They suggested the use of QR codes and information projection (or tablets) as means for personalized information delivery to museum visitors.

Manielo and Amoreti [6] suggest an AR system for indoors sites to easily share online $3 \mathrm{~d}$ models and create virtual galleries at the original place. Since a large amount of $\mathrm{CH}$ data, which are not used for anything real, are merely available on web and not directly in situ, they propose to use them in order to achieve a thematic collection, printing in $3 \mathrm{~d}$ the collections when the original archaeological pieces are not disposable. They also propose the use of augmented reality, intended as video mapping, for the visualization of the reconstructed artwork. This is an entirely open source process, which can provide new perspectives of study, preservation and use, and see in this kind of temporary collections a way not to replace the good, but to strengthen it in its communicative aspect. This technique allows the visitor to be able to see archaeological artworks normally not visible because situated in other collections or museums. The use of a video mapping performance on a perfect 3D printed copy underlines the great perspectives in museum communication and didactic.

Liestøl. [4] presented an outdoors mixed reality system. In order to provide highly visual interfaces, close to the future real time 3D, they have created a static (frozen) 3D version of the current environment, based on photogrammetry, in order to test how it may play a role as an intermediate level between the real present and reconstructed past. Liestøl presents the experiences they have gained so far exploring this combination on location at the archaeological site Ancient Phalasarna on Crete, while they are waiting for the real time $3 \mathrm{D}$ to emerge as a promising alternative to current solutions.

Mokatren and Kuflik [8] examined the potential of using a mobile eye tracker for indoor positioning and intuitive interaction. In particular they work explores the potential of mobile eye-tracking and vision technology in enhancing the museum visit experience. Their hypothesis is that the use of the eye tracking technology in museums' mobile guides can enhance the visit experience by enabling more intuitive interaction. They report on satisfactory preliminary results from examining the performance of a mobile eye tracker in a realistic setting - the technology has reached a reliable degree of maturity that can be used for developing a system based on it.

Starting from a collaboration with a worldwide famous Italian designer, Calandra et al. [2] defined a Natural User Interface (NUI) to explore $360^{\circ}$ panoramic artworks presented on wallsized displays. Specifically, they let the user to "move the head" as way of natural interaction to explore these large digital artworks. To this aim, they developed a system including a remote head pose estimator to catch movements of users standing in front of the wall-sized display. With NUIs, it is difficult to get feedbacks from the users about the interest for the point of the artwork he/she is looking at. To solve this issue, they complemented the gaze estimator with a preliminary emotional analysis solution, able to implicitly infer the interest of the user for the shown content from his/her pupil size. Preliminary results on a sample of 51 subjects show that the most of the subjects were able to properly interact with the system from the very first use, and that the emotional module is an interesting solution, even if further work must be devoted to address specific situations.

Rapp et al. [12] present a reward-based field evaluation of the interaction model developed for Wanteat, an intelligent mobile application in food domain, which puts together real and virtual words. Since real objects are seen as gateways for accessing the cultural heritage of a territory, they designed an intelligent interaction model that allows users to explore the world starting from a contacted object. In particular the interaction model support the visualization and the exploration of identifiable objects of the real world and their connections with other objects. The interaction model proposes a paradigm that enables a personalized, social and serendipitous interaction with networked things, allowing a continuous transition between real and digital world. They illustrate the procedure and the results of such evaluation, carried out with a prototype application with no active users community. Results show that the interaction model stimulates the exploration of the objects in the system and their networks, and partially promotes the interactive features of the application, as social actions.

Origlia et al. [9] present a human-robot interaction setup where people actively choose how much information concerning the available topics they would like to access. To provide engaging presentations, a humanoid robot exhibiting a general behaviour modelled on the basis of a human presenter was used and a mathematical model to keep track of content navigation was designed. Monitoring the evolution of the interactive session allows estimating users' general interest towards the available contents. The results show that people were very satisfied by the interaction experience and that interests detected automatically by the system were found to be consistent with the users'. Both subjective and objective metrics were used to validate the approach.

Finally, Orio [10] reports the results of an initial experiment on the acoustic description, called soundscape, of the city of Padova. A group of users has been involved in recording the sounds of the city and in tracking their position in space and in time using a web based interface. Collaboration and coordination among participants has been promoted using a wiki, where participants could assign themselves the locations to be recorded and define the standard to be followed. The result is the creation of an acoustic map of the city of Padova, which can be navigated in space and in time through a web interface. A mobile version of the interface is under development. 


\subsection{Online interaction}

There were a few online-only systems, again, quite diverse. Lanir et al. [7] present a system that visualizes visitors' behavior onsite to museum director. They use automatic tracking of visitors' position and movements at the museum to $\log$ visitor information. Using this information, they provide an interface that visualizes both individual and small group movement patterns, as well as aggregated information of overall visitor engagement.

Liestøl [5] presents novel ideas for using storytelling for accessing online databases. In the paper he presents the exploitation of narrative theory in order to discover and develop a design, and further implement the solution in a reconstruction and simulation of an historical battle scene from World War II.

Finally Wecker et al. [14] brings up the idea of ongoing events collection - a "scrapbook" for future use. They present the idea concerning the utility and makeup of an application to collect cultural heritage experiences. They take a short look at what has been done in this area both from a lifelong cultural heritage perspective and that of timelines and memory logging visualization. The features for such an application and possible visualizations are discussed.

\subsection{Interaction techniques and technologies}

As far as technologies, the diverse set of papers spans a large variety of presentation and interaction techniques. Including conventional desktop display [7], through mobile guides [12][4], eye tracking [8][2], augmented/mixed reality [4][6], virtual reality $[11][2][3]$, natural language interaction [13], audio [10], robot interaction [9], storytelling [5].

\section{SUMMARY}

The submissions to the workshop demonstrate the wide diversity of aspects and as a results the technologies and their combination that are applied and experimented with in cultural heritage. The rich information that is available and the need to deliver it in compact and attractive manner continue to draw research attention and practically, any novel technology is being experimented with in this area.

\section{REFERENCES}

[1] https://www.google.com/culturalinstitute/project/artproject

[2] Davide Maria Calandra, Dario Di Mauro, Franco Cutugno and Sergio Di Martino (2016). Navigating Wall-sized Displays with the Gaze: a Proposal for Cultural Heritage, $A V I^{*} \mathrm{CH}$, the first Workshop on Advanced Visual Interfaces for Cultural Heritage, in conjunction with AVI 2016, the International Working Conference on Advanced Visual Interfaces, Bari, 7-10 June 2016.

[3] Fabio Marco Caputo, Irina Mihaela Ciortan, Davide Corsi, Marco De Stefani and Andrea Giachetti. (2016). Gestural Interaction and Navigation Techniques Aimed for Virtual Museum Experiences, $A V I^{*} C H$, the first Workshop on Advanced Visual Interfaces for Cultural Heritage, in conjunction with AVI 2016, the International
Working Conference on Advanced Visual Interfaces, Bari, 7-10 June 2016.

[4] Gunnar Liestøl. (2016a). Quasi-Mixed Reality in Cultural Heritage. Combining the present with the past in situ, $A V I^{*} \mathrm{CH}$, the first Workshop on Advanced Visual Interfaces for Cultural Heritage, in conjunction with AVI 2016, the International Working Conference on Advanced Visual Interfaces, Bari, 7-10 June 2016.

[5] Gunnar Liestøl. (2016b). Sequence \& Access (Discourse $\&$ Digression). Storytelling, narrative theory and access to online archives and databases when reconstructing a historic scene for display on location, $A V I * C H$, the first Workshop on Advanced Visual Interfaces for Cultural Heritage, in conjunction with AVI 2016, the International Working Conference on Advanced Visual Interfaces, Bari, 7-10 June 2016.

[6] Donato Maniello and Valeria Amoretti. (2016). Handle digital data to turn them into immersive experience using the augmented reality, $A V I^{*} \mathrm{CH}$, the first Workshop on Advanced Visual Interfaces for Cultural Heritage, in conjunction with AVI 2016, the International Working Conference on Advanced Visual Interfaces, Bari, 7-10 June 2016.

[7] Joel Lanir, Tsvi Kuflik, Nisan Yavin, Kate Leiderman and Michael Segal. (2016) Visualizing Museum Visitors' Behavior, $A V I * C H$, the first Workshop on Advanced Visual Interfaces for Cultural Heritage, in conjunction with AVI 2016, the International Working Conference on Advanced Visual Interfaces, Bari, 7-10 June 2016.

[8] Moayad Mokatren and Tsvi Kuflik (2016). Exploring the potential contribution of mobile eye-tracking technology in enhancing the museum visit experience, $A V I^{*} \mathrm{CH}$, the first Workshop on Advanced Visual Interfaces for Cultural Heritage, in conjunction with AVI 2016, the International Working Conference on Advanced Visual Interfaces, Bari, 7-10 June 2016.

[9] Antonio Origlia, Antonio Rossi, Maria Laura Chiacchio and Francesco Cutugno. (2016). Cultural heritage presentations with a humanoid robot using implicit feedback, $A V I * C H$, the first Workshop on Advanced Visual Interfaces for Cultural Heritage, in conjunction with AVI 2016, the International Working Conference on Advanced Visual Interfaces, Bari, 7-10 June 2016.

[10] Nicola Orio. (2016). Padova Soundscape: a Crowdsourcing Approach to Describe the Sound of a City

[11] Nikolaos Partarakis, Margherita Antona, Constantine Stephanidis and Emmanouil Zidianakis. (2016). Adaptation and content personalization in the context of multi user museum exhibits, $A V I^{*} \mathrm{CH}$, the first Workshop on Advanced Visual Interfaces for Cultural Heritage, in conjunction with AVI 2016, the International Working Conference on Advanced Visual Interfaces, Bari, 7-10 June 2016.

[12] Amon Rapp, Federica Cena, Luca Console, Cristina Gena and Alessandro Marcengo. (2016). A Field Evaluation of an Intelligent Interaction Between People and a Territory and its Cultural Heritage, $A V I^{*} C H$, the first Workshop on 
Advanced Visual Interfaces for Cultural Heritage, in conjunction with AVI 2016, the International Working Conference on Advanced Visual Interfaces, Bari, 7-10 June 2016.

[13] Antonio Sorgente, Paolo Vanacore, Antonio Origlia, Enrico Leone, Francesco Cutugno and Francesco Mele. (2016). Multimedia Responses in Natural Language Dialogues, $A V I^{*} \mathrm{CH}$, the first Workshop on Advanced Visual Interfaces for Cultural Heritage, in conjunction with AVI 2016, the International Working Conference on Advanced Visual Interfaces, Bari, 7-10 June 2016.

[14] Alan Wecker, Tsvi Kuflik and Oliviero Stock. (2016). CHEST: Cultural Heritage Experience Scrapbook Tool, $A V I^{*} \mathrm{CH}$, the first Workshop on Advanced Visual Interfaces for Cultural Heritage, in conjunction with AVI 2016, the International Working Conference on Advanced Visual Interfaces, Bari, 7-10 June 2016. 